\title{
Re-classification of ROTSE-I $\delta$ Scuti stars
}

\author{
H. Jin, S.-L. Kim, C.-U. Lee, D.-J. Lee \\ Korea Astronomy Observatory, Daejeon, 305-348, Korea \\ K.-S. Kim \\ Department of Astronomy and Space Science, Kyunghee University, \\ Yongin, 449-701, Korea
}

\begin{abstract}
We present new multiband photometric results for ROTSE-I $\delta$ Sct stars and the Fourier decomposition analysis. Our result shows that most of the stars classified as ROTSE-I $\delta$ Sct stars seem to be W UMa type eclipsing variable stars.
\end{abstract}

\section{Introduction}

In a previous study we showed that a number of ROTSE-I $\delta$ Sct type stars, which do not show typical pulsating light curves of high amplitude $\delta$ Sct stars, are in fact W UMa type eclipsing binaries (Jin et al. 2003). To distinguish between eclipsing and pulsating stars, we used the criterion that pulsating stars have larger amplitude differences between passbands than eclipsing binaries do because brightness changes of pulsating stars are mainly due to the temperature variations.

\section{Observations and reductions}

We performed new follow-up observations of additional 28 ROTSE-I $\delta$ Sct variables. CCD photometric observations were carried out on 32 nights from 2003 March to June. We obtained time series CCD images with $B, V$ and $I$ filters with a $1 \mathrm{~K}$ Apogee CCD camera attached to the KAO 1-m telescope installed at Mt. Lemmon, Arizona, USA. We processed the CCD images to subtract bias frames and correct the pixel-to-pixel inhomogeneity of quantum efficiency, flat fielding. Instrumental magnitudes were obtained via the simple aperture photometric routine in the IRAF/APPHOT package. We calculated differential magnitudes of each variable star using a nearby comparison star with similar brightness and color to the variable. Fig. 1 shows a sample figure of the photometric result.

\section{Results and conclusions}

We found that 26 objects among 29 target stars turned out to be W UMa type eclipsing binaries. Fig. 2 displays the amplitude differences of eclipsing binaries 

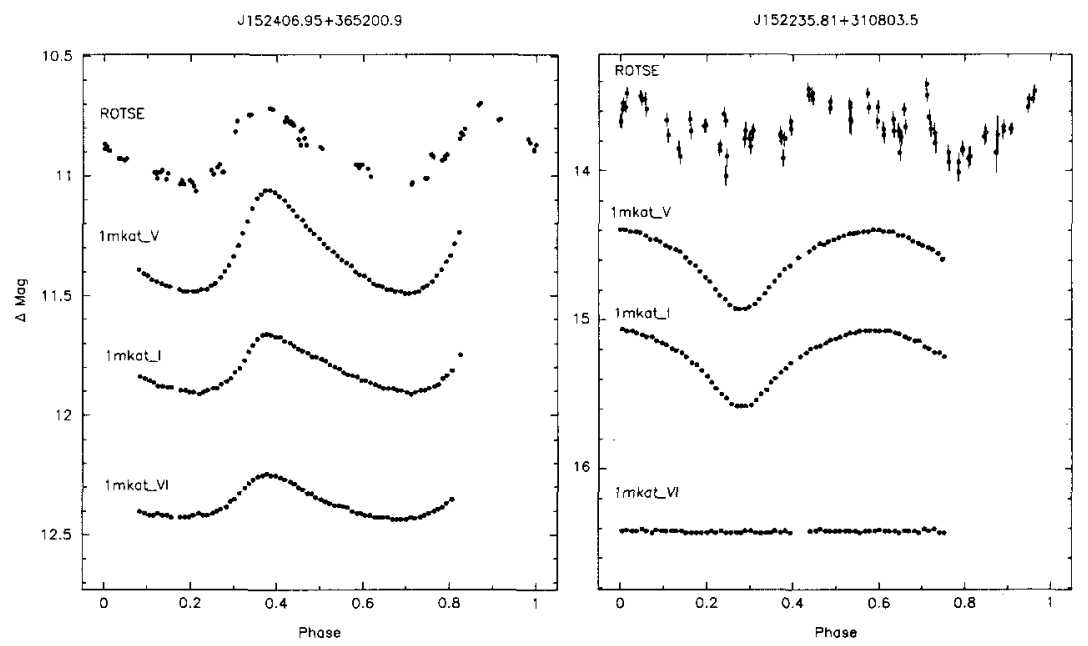

Figure 1. Sample light curves of a pulsating star (left) and an eclipsing binary (right) obtained with the KAO 1-m telescope at Mt. Lemmon. Mean magnitudes of each data set are expressed on an arbitrary scale.

as well as pulsating stars between two passbands, $V$ and $I$. The slopes of $\Delta I$ against $\Delta V$ are about 0.92 for $\mathrm{W}$ UMa stars, but 0.62 for pulsating stars. This large difference indicates that the light variation mechanism of pulsating stars is quite different from that of eclipsing variables: brightness changes of pulsating stars are mainly due to the temperature variations. The amplitudes of pulsating stars decrease rapidly in the infrared passband.

We applied Fourier decomposition analysis to the ROTSE-I $\delta$ Sct type stars to find an effective classification method in survey projects (see Poretti 2001; Morgan 2003). Fig. 3 shows the $\emptyset_{21}$ versus period diagram for the $I$ band magnitude of our photometric results and the ROTSE-I data. We find a separation between W UMa eclipsing binaries and $\delta$ Sct pulsating stars. The $\emptyset_{21}$ of eclipsing variable stars have a tendency toward 6 or 0 , because most of W UMa type eclipsing binaries have symmetric light curves. On the other hand, those of pulsating stars concentrate around 4. More detailed results will be presented in another paper. As our study of re-classification has shown, the ROTSE-I $\delta$ Sct stars need an inspection such as Fourier decomposition analysis and a follow-up observations with multiband photometry to classify each star clearly.

\section{References}

Jin, H. et al. 2003, A\&A, 404, 621

Morgan, S.M. 2003, PASP, 115, 1250

Poretti, E. 2001, A\&A, 371, 986 


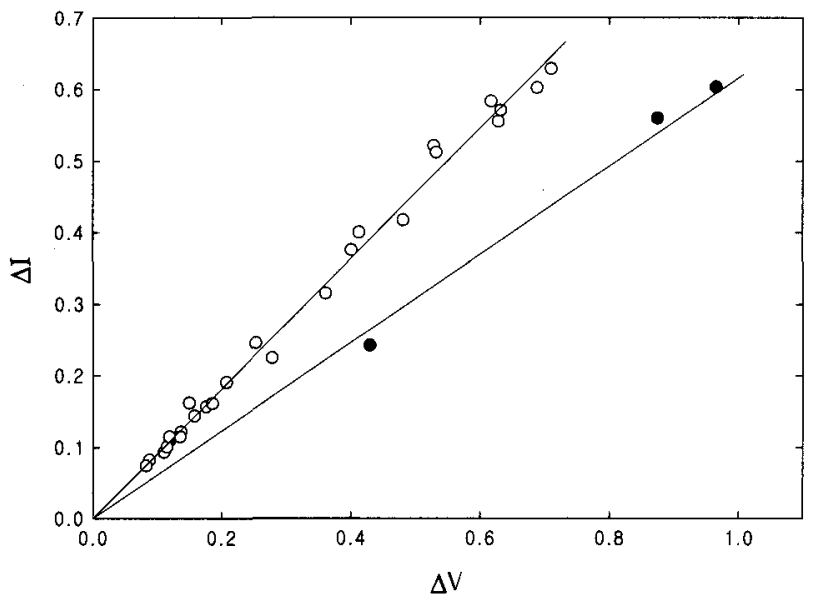

Figure 2. $\Delta V$ versus $\Delta I$ amplitude difference diagram. Open circles represent W UMa type eclipsing binaries and filled circles denote pulsating stars. The slopes of $\Delta I$ against $\Delta V$ are very different between the types of two variables.

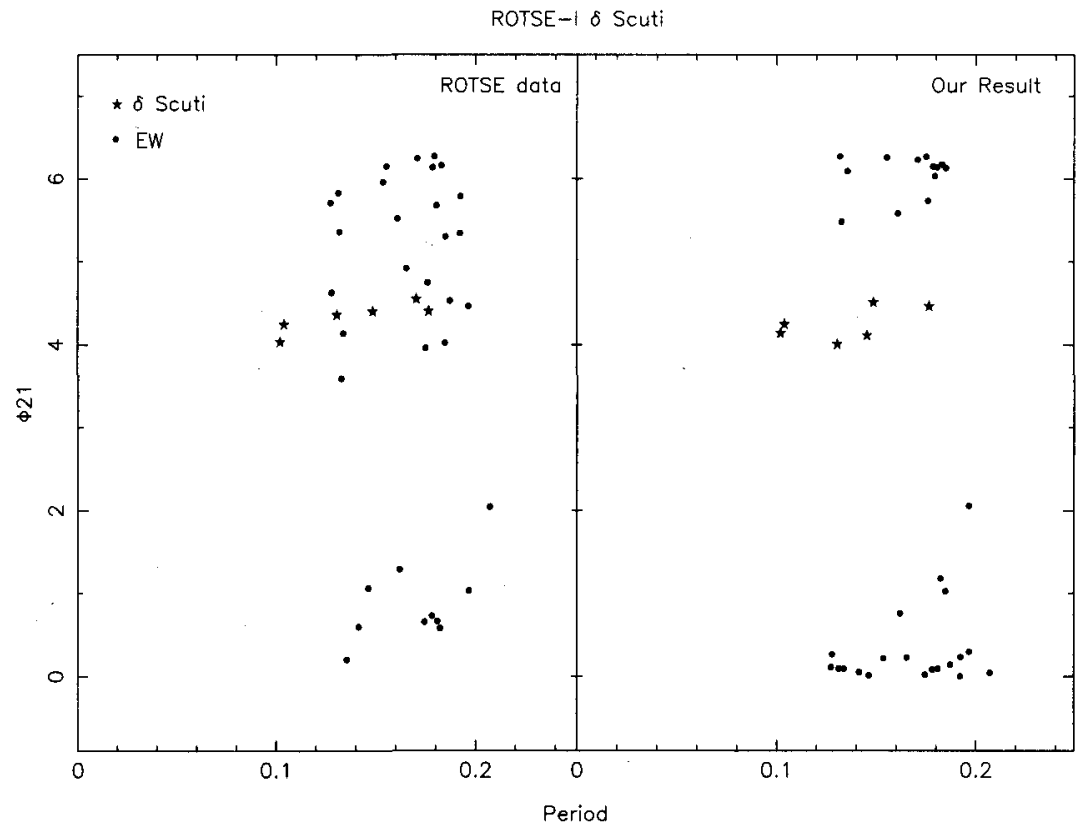

Figure 3. The Fourier coefficient $\emptyset_{21}$ against period for our total 39 follow-up observing targets of ROTSE-I $\delta$ Sct type stars. The left panel uses the ROTSE-I data and the right one uses our $I$-band data. The separation between W UMa eclipsing variables and $\delta$ Sct stars is clearer in the right panel. 


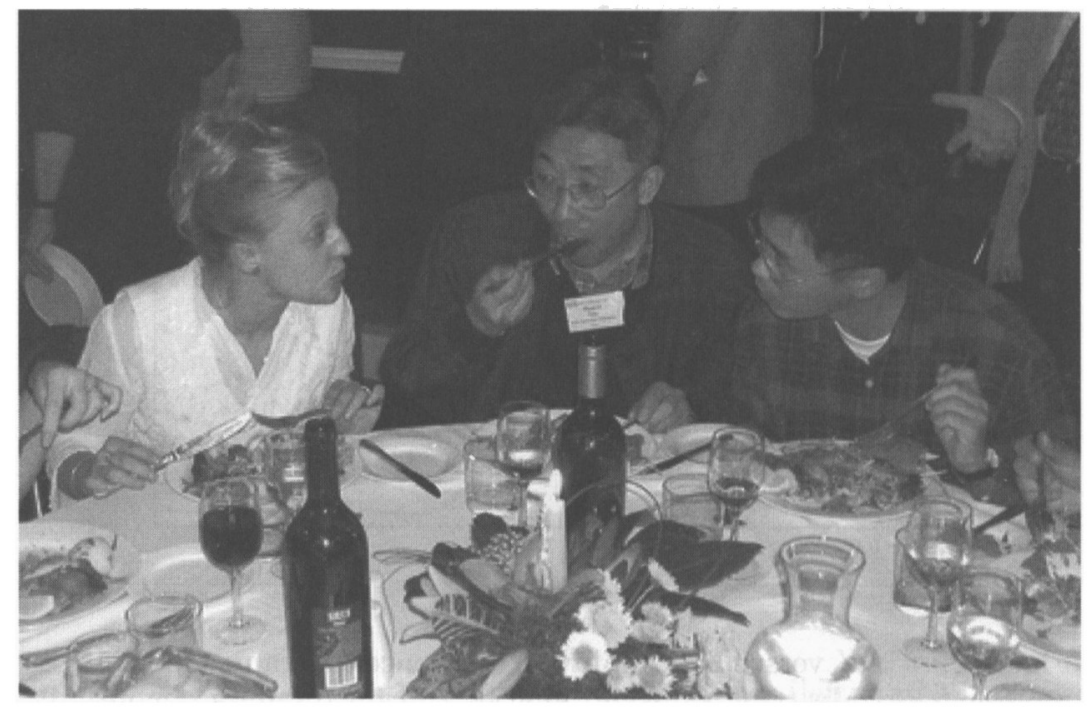

Jagoda Daszyńska-Daszkiewicz, Hyun-il Sung and Ho Jin

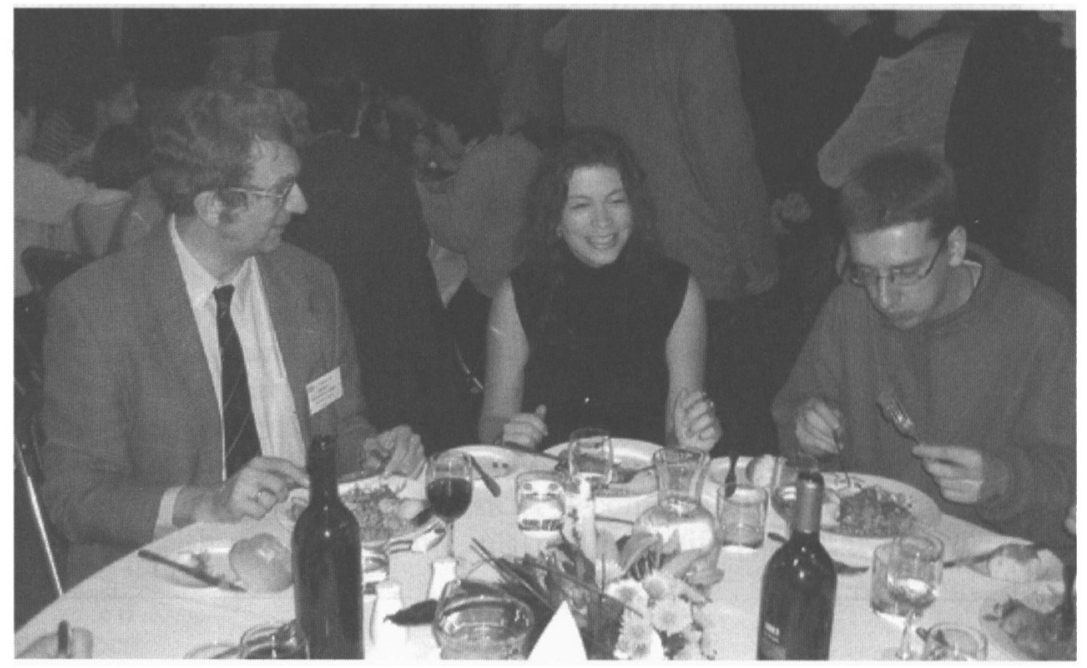

Jørgen Christensen-Dalsgaard, Caroline Schoenaers and Joris De Ridder 